\title{
Deformed higher rank Heisenberg-Virasoro algebras
}

\author{
Chengkang $\mathrm{Xu} 1$ \\ Shangrao Normal University, Shangrao, Jiangxi, China \\ Email: xiaoxiongxu@126.com
}

\begin{abstract}
In this paper, we study a class of infinitesimal deformations of the centerless higher rank Heisenberg-Virasoro algebras. Explicitly, the universal central extensions, derivations and isomorphism classes of these algebras are determined.

Keywords: Heisenberg-Virasoro algebra, deformed Heisenberg-Virasoro algebra, higher rank Heisenberg-Virasoro algebra, infinitesimal deformation.

MSC(2010): 17B05, 17B40, 17B56, $17 \mathrm{~B} 68$.
\end{abstract}

\section{Introduction}

Let $G$ be an additive subgroup of $\mathbb{C}$ and $\lambda \in \mathbb{C}$. The Lie algebra $\mathfrak{g}(G, \lambda)$ we study in this paper has a basis $\left\{L_{a}, I_{a} \mid a \in G\right\}$ subjecting to the following Lie brackets

$$
\left[L_{a}, L_{b}\right]=(b-a) L_{a+b} ; \quad\left[L_{a}, I_{b}\right]=(b-\lambda a) I_{a+b} ; \quad\left[I_{a}, I_{b}\right]=0 .
$$

We will simply denote $\mathfrak{g}=\mathfrak{g}(G, \lambda)$ if no confusion. Since for any nonzero $\epsilon \in \mathbb{C}$, there is a Lie algebra isomorphism between $\mathfrak{g}(G, \lambda)$ and $\mathfrak{g}(\epsilon G, \lambda)$ defined by

$$
L_{a} \mapsto \epsilon^{-1} L_{\epsilon a} ; \quad I_{a} \mapsto \epsilon^{-1} I_{\epsilon a},
$$

we always assume in this paper that $\mathbb{Z} \subseteq G$ and $\frac{1}{k} \notin G$ for any integer $k>1$.

The algebra $\mathfrak{g}$ is formed through semi-product of the higher rank Virasoro algebra $\mathfrak{v}=\operatorname{span}_{\mathbb{C}}\left\{L_{a} \mid a \in G\right\}$ and one of its module of tensor fields $V(\lambda)=\operatorname{span}_{\mathbb{C}}\left\{v_{a} \mid a \in G\right\}$ defined by

$$
L_{a} v_{b}=(b-\lambda a) v_{a+b} .
$$

Notice that the algebra $\mathfrak{g}(G, 0)$ is the centerless higher rank Heisenberg-Virasoro algebra [8], and $\mathfrak{g}(G, \lambda)$ is an infinitesimal deformation of $\mathfrak{g}(G, 0)$. Therefore, we call $\mathfrak{g}(G, \lambda)$ a centerless deformed higher rank Heisenberg-Virasoro algebra. It also has close connections with the Heisenberg-Virasoro algebra and deformed Heisenberg-Virasoro algebras. Taking $G=\mathbb{Z}$, we get the centerless deformed Heisenberg-Virasoro algebra $\mathfrak{g}(\mathbb{Z}, \lambda)$, which was given in [7] through the second cohomology group of the centerless HeisenbergVirasoro algebra $\mathfrak{g}(\mathbb{Z}, \lambda)$ with coefficients in the adjoint representation. Hence the deformed Heisenberg-Virasoro algebras and higher rank Heisenberg-Virasoro algebras are generalizations of the Heisenberg-Virasoro algebra, which was first introduced in [1]. Representations of these three algebras were studied in [5, 6, 9] and references therein.

\footnotetext{
1 The author is supported by the National Natural Science Foundation of China (No. 11801375)
} 
Due to the connection with these algebras, it seems reasonable and important to study the algebra $\mathfrak{g}$. Our main purpose of this paper is to determine the universal central extensions, derivations and isomorphism classes of all $\mathfrak{g}(G, \lambda)$. Such computation is a priority in the structure theory of Lie algebra, and has been done for many Lie algebras, such as generalized Witt algebras [3, generalized Schrodinger-Virasoro algebras [13, 12, higher rank Heisenberg-Virasoro algebras [8], deformed Heisenberg-Virasoro algebras [7], solenoidal Lie algebras over a quantum torus [14], and so on.

The following is the outline of this paper. In section 2, we compute the universal central extension $\overline{\mathfrak{g}}$ of $\mathfrak{g}$, which we call deformed higher rank Heisenberg-Virasoro algebra. Section 3 is devoted to derivations of $\mathfrak{g}$ and the lifts of these derivations to derivations of $\overline{\mathfrak{g}}$. In the last section isomorphism classes and automorphism groups of all the algebras $\mathfrak{g}(G, \lambda)$ are determined, and finally the explicit form of the unique lift of any outer automorphism of $\mathfrak{g}$ to $\overline{\mathfrak{g}}$ is given.

Throughout this paper, the symbols $\mathbb{Z}, \mathbb{C}, \mathbb{C}^{\times}$refer to the set of integers, complex numbers and nonzero complex numbers respectively. We denote by $G^{*}$ the set of characters of $G$, and by $\operatorname{Hom}(G, \mathbb{C})$ the set of all additive group homomorphisms.

\section{Universal central extensions}

In this section we compute the universal central extensions of $\mathfrak{g}$ for $\lambda \neq-1, G$ being free and of rank $n \geq 1$. We fix a $\mathbb{Z}$-basis $\epsilon_{1}=1, \epsilon_{2}, \cdots, \epsilon_{n}$ of $G$.

Let $\varphi: \mathfrak{g} \times \mathfrak{g} \longrightarrow \mathbb{C}$ be a 2-cocycle, hence

$$
\begin{aligned}
& \varphi([x, y], z)+\varphi([y, z], x)+\varphi([z, x], y)=0 \\
& \varphi(x, y)=-\varphi(y, x), \quad \text { for any } x, y, z \in \mathfrak{g} .
\end{aligned}
$$

Define a linear function $f$ on $\mathfrak{g}$ by

$$
\begin{aligned}
& f\left(L_{a}\right)=\frac{1}{a} \varphi\left(L_{0}, L_{a}\right) ; \quad f\left(I_{a}\right)=\frac{1}{a} \varphi\left(L_{0}, I_{a}\right) \text { for } a \neq 0 ; \\
& f\left(L_{0}\right)=\frac{1}{2} \varphi\left(L_{-1}, L_{1}\right) ; \quad f\left(I_{0}\right)=\frac{1}{\lambda+1} \varphi\left(L_{-1}, I_{1}\right),
\end{aligned}
$$

and a 2 -coboundary $\varphi_{f}$ by $\varphi_{f}(x, y)=f([x, y])$. Replacing $\varphi$ by $\varphi-\varphi_{f}$, we may assume

$$
\varphi\left(L_{0}, L_{a}\right)=\varphi\left(L_{0}, I_{a}\right)=\varphi\left(L_{-1}, L_{1}\right)=\varphi\left(L_{-1}, I_{1}\right)=0 \quad \text { for any } a \neq 0 \text {. }
$$

Lemma 2.1. $\varphi\left(L_{a}, L_{b}\right)=\frac{1}{12}\left(a^{3}-a\right) C_{L} \delta_{a+b, 0}$ for some $C_{L} \in \mathbb{C}$.

Proof. One reference for this computation is [11].

Lemma 2.2. $\varphi\left(I_{a}, I_{b}\right)=a C_{I} \delta_{a+b, 0} \delta_{\lambda, 0}$ for some $C_{I} \in \mathbb{C}$.

Proof. Consider the triple $(x, y, z)=\left(L_{a}, I_{b}, I_{c}\right)$ in (2.1) and we have

$$
(b-\lambda a) \varphi\left(I_{a+b}, I_{c}\right)+(c-\lambda a) \varphi\left(I_{b}, I_{a+c}\right)=0 .
$$


Set $a=0$ in (2.2) and we get

$$
\varphi\left(I_{b}, I_{c}\right)=0 \text { if } b+c \neq 0 .
$$

Take $c=-a-b$ in $(\underline{2.2})$,

$$
(b-\lambda a) \varphi\left(I_{a+b}, I_{-a-b}\right)=((\lambda+1) a+b) \varphi\left(I_{b}, I_{-b}\right) .
$$

Set $a=1-b$ in (2.3), we obtain

$$
-(\lambda b-\lambda-1) \varphi\left(I_{b}, I_{-b}\right)=((\lambda+1) b-\lambda) \varphi\left(I_{1}, I_{-1}\right),
$$

which implies the lemma for $\lambda=0$ by setting $C_{I}=\varphi\left(I_{1}, I_{-1}\right)$.

Now we only need to prove that $\varphi\left(I_{a}, I_{-a}\right)=0$ for any $a \in G$ if $\lambda \neq 0,-1$. If $\lambda^{-1} \in \mathbb{Z}$, then set $a=\frac{b}{\lambda} \in G$ in $(2.3)$ and one gets

$$
b\left(\frac{1}{\lambda}+2\right) \varphi\left(I_{b}, I_{-b}\right)=0
$$

which shows that $\varphi\left(I_{b}, I_{-b}\right)=0$ if $\lambda \neq-\frac{1}{2}$. Suppose $\lambda=-\frac{1}{2}$ and by (2.4) we see

$$
\varphi\left(I_{b}, I_{-b}\right)=\varphi\left(I_{1}, I_{-1}\right) \text { if } b \neq-1 .
$$

Choose $b \neq \pm 1$ and let $a=-1-b$ in (2.3), we get

$$
\frac{b-1}{2} \varphi\left(I_{-1}, I_{1}\right)=\frac{b-1}{2} \varphi\left(I_{b}, I_{-b}\right)=\frac{b-1}{2} \varphi\left(I_{1}, I_{-1}\right)
$$

which forces that $\varphi\left(I_{1}, I_{-1}\right)=0$ by skew-symmetry. So $\varphi\left(I_{b}, I_{-b}\right)=0$.

Suppose that $\lambda^{-1} \notin \mathbb{Z}$, then $\lambda b+\lambda+1 \neq 0$ for any $b \in G$. So (2.4) gives

$$
\varphi\left(I_{a}, I_{-a}\right)=-\frac{(\lambda+1) a-\lambda}{\lambda a-\lambda-1} \varphi\left(I_{1}, I_{-1}\right) .
$$

Put it into (2.3) and set $b=0$, then we get

$$
\lambda(\lambda+1)(2 \lambda+1) a(a-1) \varphi\left(I_{1}, I_{-1}\right)=0 \text { for any } a \in G,
$$

which forces $\varphi\left(I_{1}, I_{-1}\right)=0$, and hence $\varphi\left(I_{a}, I_{-a}\right)=0$. We complete the proof.

Lemma 2.3. $\varphi\left(L_{a}, I_{b}\right)=\delta_{a+b, 0}\left(C_{L I}^{(0)}\left(a^{2}+a\right) \delta_{\lambda, 0}+\frac{1}{12}\left(a^{3}-a\right) C_{L I}^{(1)} \delta_{\lambda, 1}+\sum_{i=2}^{n} a_{i} C_{L I}^{(i)} \delta_{\lambda,-2}\right)$, where $C_{L I}^{(i)} \in \mathbb{C}, 0 \leq i \leq n$ and $a=\sum_{j=1}^{n} a_{j} \epsilon_{j}$.

Proof. Consider the triple $(x, y, z)=\left(L_{a}, L_{b}, I_{c}\right)$ in (2.1), we have

$$
(b-a) \varphi\left(L_{a+b}, I_{c}\right)-(c-\lambda b) \varphi\left(L_{a}, I_{b+c}\right)+(c-\lambda a) \varphi\left(L_{b}, I_{a+c}\right)=0 .
$$


Take $a=0$,

$$
(b+c) \varphi\left(L_{b}, I_{c}\right)-(c-\lambda b) \varphi\left(L_{0}, I_{b+c}\right)=0,
$$

which implies

$$
\varphi\left(L_{b}, I_{c}\right)=0 \text { if } b+c \neq 0 .
$$

Moreover, let $b+c=0$ and $c-\lambda b \neq 0($ such $b, c$ exist since $\lambda \neq-1)$, we get $\varphi\left(L_{0}, I_{0}\right)=0$.

Set $c=-a-b$ in (2.5) and we obtain

$$
(b-a) \varphi\left(L_{a+b}, I_{-a-b}\right)+(a+b(\lambda+1)) \varphi\left(L_{a}, I_{-a}\right)-(a(\lambda+1)+b) \varphi\left(L_{b}, I_{-b}\right)=0 .
$$

Let $a+b=0$ in (2.7) and we get

$$
\varphi\left(L_{-a}, I_{a}\right)=-\varphi\left(L_{a}, I_{-a}\right) \text { if } \lambda \neq 0 .
$$

Let $b=1$ in (2.7), we have

$$
(1-a) \varphi\left(L_{a+1}, I_{-a-1}\right)+(a+\lambda+1) \varphi\left(L_{a}, I_{-a}\right)-(a(\lambda+1)+1) \varphi\left(L_{1}, I_{-1}\right)=0 .
$$

Moreover, setting $b=-1$ and replacing $a$ by $a+1$ in (2.7), it gives

$$
(a-\lambda) \varphi\left(L_{a+1}, I_{-a-1}\right)-(a+2) \varphi\left(L_{a}, I_{-a}\right)=0 .
$$

Now we continue the proof in the following four cases.

Case I: $\lambda=0$. Combining (2.9) and (2.10), we get

$$
\varphi\left(L_{a}, I_{-a}\right)=\frac{1}{2}\left(a^{2}+a\right) \varphi\left(L_{1}, I_{-1}\right) .
$$

Set $C_{L I}^{(0)}=\frac{1}{2} \varphi\left(L_{1}, I_{-1}\right)$, and we get the lemma by (2.6).

We emphasize that in the remaining three cases we have $\varphi\left(L_{-a}, I_{a}\right)=-\varphi\left(L_{a}, I_{-a}\right)$ for any $a \in G$. Especially, $\varphi\left(L_{1}, I_{-1}\right)=-\varphi\left(L_{-1}, I_{1}\right)=0$.

Case II: $\lambda \neq 0, \pm 1,-2$. Notice that (2.9) and (2.10) form a system of homogeneous liner equations in variables $\varphi\left(L_{a}, I_{-a}\right)$ and $\varphi\left(L_{a+1}, I_{-a-1}\right)$ with a coefficient matrix whose determinant is

$$
\left|\begin{array}{cc}
1-a & a+\lambda+1 \\
a-\lambda & -a-2
\end{array}\right|=(\lambda-1)(\lambda+2) \neq 0 .
$$

So $\varphi\left(L_{a}, I_{-a}\right)=0$ and hence by (2.6) $\varphi\left(L_{a}, I_{b}\right)=0$ for any $a, b \in G$.

Case III: $\lambda=1$. Set $C_{L I}^{(1)}=2 \varphi\left(L_{2}, I_{-2}\right)$. A same calculation as in Lemma 2.1 shows

$$
\varphi\left(L_{a}, I_{b}\right)=\frac{1}{12}\left(a^{3}-a\right) C_{L I}^{(1)} \delta_{a+b, 0} .
$$

Case IV: $\lambda=-2$. In this case, the equations (2.9) and (2.10) turn to

$$
\begin{aligned}
& (a-1) \varphi\left(L_{a}, I_{-a}\right)=(a-1) \varphi\left(L_{a+1}, I_{-a-1}\right), \\
& (a+2) \varphi\left(L_{a}, I_{-a}\right)=(a+2) \varphi\left(L_{a+1}, I_{-a-1}\right),
\end{aligned}
$$


which implies that $\varphi\left(L_{a+1}, I_{-a-1}\right)=\varphi\left(L_{a}, I_{-a}\right)$ for any $a \in G$. So

$$
\varphi\left(L_{k}, I_{-k}\right)=0, \quad \varphi\left(L_{a+k}, I_{-a-k}\right)=\varphi\left(L_{a}, I_{-a}\right) \text { for any } k \in \mathbb{Z}, a \in G \backslash \mathbb{Z} .
$$

Then (2.5) gives

$$
\varphi\left(L_{a+b}, I_{-a-b}\right)=\varphi\left(L_{a}, I_{-a}\right)+\varphi\left(L_{b}, I_{-b}\right) \text { if } a \neq b .
$$

For $a \in G \backslash \mathbb{Z}$, we still have

$$
\varphi\left(L_{2 a}, I_{-2 a}\right)=\varphi\left(L_{a+1}, I_{-a-1}\right)+\varphi\left(L_{a-1}, I_{-a+1}\right)=2 \varphi\left(L_{a}, I_{-a}\right) .
$$

So we have proved $\varphi\left(L_{a+b}, I_{-a-b}\right)=\varphi\left(L_{a}, I_{-a}\right)+\varphi\left(L_{b}, I_{-b}\right)$ for any $a, b \in G$. Therefore $\varphi\left(L_{a}, I_{-a}\right)=\sum_{i=2}^{n} a_{i} \varphi\left(L_{\epsilon_{i}}, I_{-\epsilon_{i}}\right)$ if $a=\sum_{i=1}^{n} a_{i} \epsilon_{i}$. Put $C_{L I}^{(i)}=\varphi\left(L_{\epsilon_{i}}, I_{-\epsilon_{i}}\right)$ for $2 \leq i \leq n$, and we see that $\varphi\left(L_{a}, I_{b}\right)=\sum_{i=2}^{n} a_{i} C_{L I}^{(i)} \delta_{a+b, 0}$. This finishes the proof.

Now from Lemma 2.1, 2.2 and 2.3 we get the main theorem of this section.

Theorem 2.4. Denote by $\overline{\mathfrak{g}}$ the universal central extension of $\mathfrak{g}(G, \lambda)$ for $\lambda \neq-1$ and $G$ be a free subgroup of $\mathbb{C}$ of rank $n$. Then $\overline{\mathfrak{g}}$ satisfies the following Lie brackets

$$
\begin{aligned}
& {\left[L_{a}, L_{b}\right]=(b-a) L_{a+b}+\frac{1}{12}\left(a^{3}-a\right) C_{L} \delta_{a+b, 0} ; \quad\left[I_{a}, I_{b}\right]=a C_{I} \delta_{a+b, 0} \delta_{\lambda, 0} ;} \\
& {\left[L_{a}, I_{b}\right]=(b-\lambda a) I_{a+b}+\delta_{a+b, 0}\left(C_{L I}^{(0)}\left(a^{2}+a\right) \delta_{\lambda, 0}+\frac{1}{12}\left(a^{3}-a\right) C_{L I}^{(1)} \delta_{\lambda, 1}+\sum_{i=2}^{n} a_{i} C_{L I}^{(i)} \delta_{\lambda,-2}\right)}
\end{aligned}
$$

where $C_{L}, C_{I}, C_{L I}^{(i)}, 0 \leq i \leq n$ are central elements and $a=\sum_{i=1}^{n} a_{i} \epsilon_{i}$

Remark: (1) If $\lambda \neq-1,-2$, Theorem 2.4 stands for arbitrary additive subgroup $G$ of $\mathbb{C}$. Especially, when $\lambda=0$, we get the generalized Heisenberg-Virasoro algebras, which was originally given in [8]. When $G=\mathbb{Z}$, we get the deformed Heisenberg-Virasoro algebra, which was studied in [7].

(2) The Lie algebra $\mathfrak{g}=\mathfrak{g}(G,-1)$ is not perfect $\left(I_{0} \notin[\mathfrak{g}, \mathfrak{g}]\right)$ and there is no universal central extension of $\mathfrak{g}$. But one can still consider the universal central extension of the derived subalgebra $\mathfrak{g}^{\prime}=[\mathfrak{g}, \mathfrak{g}]=\operatorname{span}_{\mathbb{C}}\left\{L_{a}, I_{b} \mid a \in G, b \in G \backslash\{0\}\right\}$. One can prove that the universal central extension of $\mathfrak{g}^{\prime}$ is governed by four nontrivial 2-cocycles

$$
\begin{aligned}
& C_{L}\left(L_{a}, L_{b}\right)=\frac{1}{12}\left(a^{3}-a\right) \delta_{a+b, 0} ; \quad C_{I}\left(I_{a}, I_{b}\right)=\frac{1}{a} \delta_{a+b, 0} \\
& C_{L I}\left(L_{a}, I_{b}\right)=a \delta_{a+b, 0} ; \quad C_{L I}^{\prime}\left(L_{a}, I_{b}\right)=\delta_{a+b, 0} .
\end{aligned}
$$

\section{$3 \quad$ Derivations of $\mathfrak{g}$ and $\overline{\mathfrak{g}}$}

In this section we compute derivations of $\mathfrak{g}$ for arbitrary $G$ and $\lambda$, and consider the lifts of these derivations of $\mathfrak{g}$ to derivations of $\overline{\mathfrak{g}}$. 
The algebra $\mathfrak{g}$ has a natural $G$-grading $\mathfrak{g}=\bigoplus_{a \in G} \mathfrak{g}_{a}$, where $\mathfrak{g}_{a}=\operatorname{span}_{\mathbb{C}}\left\{L_{a}, I_{a}\right\}$. From a well known result about derivations of graded Lie algebras in [4] we know that the algebra Der $\mathfrak{g}$ of derivations of $\mathfrak{g}$ is also graded by $G$, i.e, Derg $=\bigoplus_{a \in G}(\operatorname{Derg})_{a}$, where

$$
(\operatorname{Derg})_{a}=\operatorname{span}_{\mathbb{C}}\left\{\sigma \in \operatorname{Derg} \mid \sigma\left(\mathfrak{g}_{b}\right) \subseteq \mathfrak{g}_{a+b}, \forall b \in G\right\},
$$

and all outer derivations of $\mathfrak{g}$ lie in $(\operatorname{Der} \mathfrak{g})_{0}$. Now we construct some derivations of $\mathfrak{g}$ which are all of degree 0 . Define linear maps $\varphi, \psi: \mathfrak{g} \longrightarrow \mathfrak{g}$ by

$$
\varphi\left(L_{a}\right)=a I_{a}, \varphi\left(I_{a}\right)=0 ; \quad \psi\left(L_{a}\right)=0, \psi\left(I_{a}\right)=I_{a} .
$$

For $\lambda=0$, we define a linear map $\sigma_{(0)}: \mathfrak{g} \longrightarrow \mathfrak{g}$ by

$$
\sigma_{(0)}\left(L_{a}\right)=I_{a} \delta_{\lambda, 0}, \sigma_{(0)}\left(I_{a}\right)=0 .
$$

For $\lambda=-1$, we define a linear map $\sigma_{(-1)}: \mathfrak{g} \longrightarrow \mathfrak{g}$ by

$$
\sigma_{(-1)}\left(L_{a}\right)=a^{2} I_{a} \delta_{\lambda,-1}, \sigma_{(-1)}\left(I_{a}\right)=0 .
$$

For $\lambda=-2$, we define a linear map $\sigma_{(-2)}: \mathfrak{g} \longrightarrow \mathfrak{g}$ by

$$
\sigma_{(-2)}\left(L_{a}\right)=a^{3} I_{a} \delta_{\lambda,-2}, \sigma_{(-2)}\left(I_{a}\right)=0 .
$$

Let $\mathcal{A} \in \operatorname{Hom}(G, \mathbb{C})$. We define a linear map $\xi_{\mathcal{A}}: \mathfrak{g} \longrightarrow \mathfrak{g}$ by

$$
\xi_{\mathcal{A}}\left(L_{a}\right)=\mathcal{A}(a) L_{a}, \xi_{\mathcal{A}}\left(I_{a}\right)=\mathcal{A}(a) I_{a},
$$

and for $\lambda=1$, a linear map $\eta_{\mathcal{A}, 1}: \mathfrak{g} \longrightarrow \mathfrak{g}$ by

$$
\eta_{\mathcal{A}, 1}\left(L_{a}\right)=\mathcal{A}(a) I_{a} \delta_{\lambda, 1}, \eta_{\mathcal{A}, 1}\left(I_{a}\right)=0 .
$$

It is easy to check that the linear maps $\varphi, \psi, \sigma_{(0)}, \sigma_{(-1)}, \sigma_{(-2)}, \xi_{\mathcal{A}}, \eta_{\mathcal{A}, 1}$ are all derivations of degree 0 . The main result of this section is the following

Theorem 3.1. For $a \neq 0,(\text { Derg })_{a}=\operatorname{span}_{\mathbb{C}}\left\{a d L_{a}, a d I_{a}\right\}$, and

$$
(\operatorname{Derg})_{0}=\operatorname{span}_{\mathbb{C}}\left\{\varphi, \psi, \sigma_{(0)}, \sigma_{(-1)}, \sigma_{(-2)}, \xi_{\mathcal{A}}, \eta_{\mathcal{A}, 1} \mid \mathcal{A} \in \operatorname{Hom}(G, \mathbb{C})\right\} .
$$

Proof. Let $\sigma \in(\text { Derg })_{0}$ and suppose

$$
\sigma\left(L_{a}\right)=\mu_{L}(a) L_{a}+\tau_{L}(a) I_{a}, \quad \sigma\left(I_{a}\right)=\mu_{I}(a) L_{a}+\tau_{I}(a) I_{a},
$$

for some functions $\mu_{L}, \tau_{L}, \mu_{I}, \tau_{I}$ on $G$. Apply $\sigma$ to $\left[L_{a}, L_{b}\right],\left[L_{a}, I_{b}\right]$ and $\left[I_{a}, I_{b}\right]$, we get the following equations

$$
\begin{aligned}
& \mu_{I}(a)(b-\lambda a)=\mu_{I}(b)(a-\lambda b), \\
& \mu_{I}(a+b)(b-\lambda a)=\mu_{I}(b)(b-a), \\
& (b-\lambda a) \tau_{I}(a+b)=(b-\lambda a) \mu_{L}(a)+(b-\lambda a) \tau_{I}(b),
\end{aligned}
$$




$$
\begin{aligned}
& (b-a) \tau_{L}(a+b)=(b-\lambda a) \tau_{L}(b)-(a-\lambda b) \tau_{L}(a) \\
& (b-a) \mu_{L}(a+b)=(b-a)\left(\mu_{L}(a)+\mu_{L}(b)\right) .
\end{aligned}
$$

Claim 1: $\mu_{L} \in \operatorname{Hom}(G, \mathbb{C})$. From (3.5) we see that $\mu_{L}(a+b)=\mu_{L}(a)+\mu_{L}(b)$ if $a \neq b$. Moreover, let $a=0, b \neq 0$ in (3.5) we get $\mu_{L}(0)=0$. Hence $\mu_{L}(-a)=-\mu_{L}(a)$ for any $a \in G$. Then choose $b \neq 0, \pm a$ and we have

$$
\mu_{L}(2 a)=\mu_{L}(a+b)+\mu_{L}(a-b)=2 \mu_{L}(a)+\mu_{L}(-b)+\mu_{L}(b)=2 \mu_{L}(a) .
$$

So we have proved $\mu_{L}(a+b)=\mu_{L}(a)+\mu_{L}(b)$ for any $a, b \in G$. Claim 1 stands.

Claim 2: $\mu_{I}=0$. Let $b=0$ in (3.2) we get

$$
\lambda \mu_{I}(a)=\mu_{I}(0) \text { for any } a \neq 0 .
$$

If $\lambda=0$, then $\mu_{I}(0)=0$. Take $b=-a$ in (3.2) and we have $\mu_{I}(a)=0$ for any $a \in G$. Suppose $\lambda \neq 0$, then $\mu_{I}(a)=\frac{1}{\lambda} \mu_{I}(0)$ for $a \neq 0$. Then (3.1) turns to

$$
\mu_{I}(0)(b-a)(\lambda+1)=0,
$$

which implies $\mu_{I}(0)=0$ and hence $\mu_{I}=0$ if $\lambda \neq-1$. If $\lambda=-1$, then take $a=-b \neq 0$ in (3.2) and we have $\mu_{I}(b)=0$ for any $b \neq 0$. So $\mu_{I}(0)=0$ by (3.6). This proves Claim 2 .

Claim 3: $\tau_{I}(a)=\mu_{L}(a)+\tau_{I}(0)$. If $\lambda=0$, then (3.3) shows that

$$
\tau_{I}(a+b)=\mu_{L}(a)+\tau_{I}(b) \text { for } b \neq 0 .
$$

Choose $a=-b \neq 0$ then we have

$$
\tau_{I}(0)=\mu_{L}(-b)+\tau_{I}(b)=-\mu_{L}(b)+\tau_{I}(b),
$$

that is, $\tau_{I}(b)=\mu_{L}(b)+\tau_{I}(0)$. This proves the claim for $\lambda=0$ since $\mu_{L}(0)=0$. Suppose $\lambda \neq 0$. Let $b=0, a \neq 0$ in (3.3), and Claim 3 follows.

Claim 4: $\tau_{L}(a)=l_{0} \delta_{\lambda, 0}+l_{1} a+l_{2} a^{2} \delta_{\lambda,-1}+l_{3} a^{3} \delta_{\lambda,-2}+\mathcal{B}(a) \delta_{\lambda, 1}$ for some $l_{0}, l_{1}, l_{2}, l_{3} \in \mathbb{C}$ and $\mathcal{B} \in \operatorname{Hom}(G, \mathbb{C})$. Take $a=0$ in (3.4) and we get

$$
\lambda \tau_{L}(0)=0
$$

Replacing $b$ by $-b$ and $a$ by $a+b$ in (3.4) we have

$$
(a+b(\lambda+1)) \tau_{L}(a+b)-(a+2 b) \tau_{L}(a)=-(\lambda a+b(\lambda+1)) \tau_{L}(-b) .
$$

Combining (3.4) and (3.8), and letting $b=1$, we obtain

$$
(\lambda-1)(\lambda+2) \tau_{L}(a)=(\lambda a-1)(a+\lambda+1) \tau_{L}(1)+(a-1)(\lambda a+\lambda+1) \tau_{L}(-1) .
$$

If $\lambda=0$, then we get

$$
\tau_{L}(a)=l_{1} a+l_{0} \text { with } l_{1}=\frac{1}{2}\left(\tau_{L}(1)-\tau_{L}(-1)\right), l_{0}=\frac{1}{2}\left(\tau_{L}(1)+\tau_{L}(-1)\right) .
$$


If $\lambda \neq 0$ then $\tau_{L}(0)=0$ by (3.7). Let $a+b=0$ in (3.4) and we get

$$
\tau_{L}(-a)=-\tau_{L}(a) \text { if } \lambda \neq 0,-1 .
$$

So by (3.9) we see $\tau_{L}(a)=a \tau_{L}(1)$ if $\lambda \neq 0, \pm 1,-2$.

If $\lambda=-1$, then from (3.9) it follows

$$
\tau_{L}(a)=l_{2} a^{2}+l_{1} a \text { with } l_{2}=\frac{1}{2}\left(\tau_{L}(1)+\tau_{L}(-1)\right), l_{1}=\frac{1}{2}\left(\tau_{L}(1)-\tau_{L}(-1)\right) .
$$

If $\lambda=1$, then (3.4) and (3.8) imply that

$$
\tau_{L}(a+b)=\tau_{L}(a)+\tau_{L}(b) \text { for any } a, b \in G,
$$

that is, $\tau_{L} \in \operatorname{Hom}(G, \mathbb{C})$. We may write $\tau_{L}(a)=\mathcal{B}(a)+l_{1} a$ for some $l_{1} \in \mathbb{C}$ and $\mathcal{B} \in$ $\operatorname{Hom}(G, \mathbb{C})$.

Now suppose $\lambda=-2$. Let $b=1$ in (3.4),

$$
(1-a) \tau_{L}(a+1)+(a+2) \tau_{L}(a)=(2 a+1) \tau_{L}(1)
$$

Furthermore, replace $a$ by $a+1$ and we have

$$
-a \tau_{L}(a+2)+(a+3) \tau_{L}(a+1)=(2 a+3) \tau_{L}(1) .
$$

Let $b=2$ in $(\underline{3.4})$,

$$
(a-2) \tau_{L}(a+2)-(a+4) \tau_{L}(a)=-2(a+1) \tau_{L}(2) .
$$

Then the above three equations imply that

$$
\tau_{L}(a)=l_{3} a^{3}+l_{1} a \text { with } l_{3}=\frac{1}{6}\left(\tau_{L}(2)-2 \tau_{L}(1)\right), l_{1}=\frac{1}{6}\left(8 \tau_{L}(1)-\tau_{L}(2)\right) .
$$

Combing the above cases we see that Claim 4 is valid.

In conclusion, we have

$$
\begin{aligned}
& \sigma\left(L_{a}\right)=\mathcal{A}(a) L_{a}+\left(l_{0} \delta_{\lambda, 0}+l_{1} a+l_{2} a^{2} \delta_{\lambda,-1}+l_{3} a^{3} \delta_{\lambda,-2}+\mathcal{B}(a) \delta_{\lambda, 1}\right) I_{a} ; \\
& \sigma\left(I_{a}\right)=(\mathcal{A}(a)+l) I_{a},
\end{aligned}
$$

for some $l, l_{0}, l_{1}, l_{2}, l_{3} \in \mathbb{C}$ and $\mathcal{A}, \mathcal{B} \in \operatorname{Hom}(G, \mathbb{C})$. This proves Theorem 3.1 .

Remark: (1) Denote by id : $G \longrightarrow \mathbb{C}$ be the identity map. The corresponding derivation $\xi_{\text {id }}$ is exactly the inner derivation $\operatorname{ad} L_{0}$. The inner derivation $\operatorname{ad} I_{0}=0$ if $\lambda=0$, and $\operatorname{ad} I_{0}=\frac{1}{\lambda} \varphi$ if $\lambda \neq 0$.

(2) In [8], derivations for higher rank Heisenberg-Virasoro algebras were computed. However, there is one derivation missed, the one we denoted by $\sigma_{(0)}$. The reason why they missed this derivation is that they assumed $\tau_{L}(0)=0$ out of nowhere (or in the terminology of [8] $\beta_{0}=0$, see Line 3 Page 9 in [8]).

In the following we consider lifts of the derivations of $\mathfrak{g}$ we obtained above to derivations of $\overline{\mathfrak{g}}$ with $\lambda \neq-1$ and $G$ being free of rank $n \geq 1$. We shall recall a result about derivations of the universal central extension of a perfect Lie algebra from [2]. 
Proposition $3.2([2])$. Suppose the Lie algebra $\mathcal{G}$ is perfect and denote by $\overline{\mathcal{G}}$ the universal central extension of $\mathcal{G}$. Then every derivation of $\mathcal{G}$ lifts to a derivation of $\overline{\mathcal{G}}$. Moreover, if $\mathcal{G}$ is centerless, then this lift is unique and Der $\overline{\mathcal{G}} \cong$ DerG.

Denote $\mathfrak{c}=\operatorname{span}_{\mathbb{C}}\left\{C_{L}, C_{I} \delta_{\lambda, 0}, C_{L I}^{(0)} \delta_{\lambda, 0}, C_{L I}^{(1)} \delta_{\lambda, 1}, C_{L I}^{(i)} \delta_{\lambda,-2} \mid 2 \leq i \leq n\right\}$. From Proposition 3.2 we know that if $\lambda \neq 0,-1$, every derivation of $\mathfrak{g}$ lifts uniquely to a derivation of $\overline{\mathfrak{g}}$. For any $\sigma \in$ Derg, define a linear map $\bar{\sigma}: \overline{\mathfrak{g}} \longrightarrow \overline{\mathfrak{g}}$ by

$$
\bar{\sigma}\left(L_{a}\right)=\sigma\left(L_{a}\right) ; \bar{\sigma}\left(I_{a}\right)=\sigma\left(I_{a}\right) ; \bar{\sigma}(\mathfrak{c})=0 \quad \text { for any } a \in G .
$$

Clearly, $\bar{\sigma}$ is a derivation of $\overline{\mathfrak{g}}$ and it lifts $\sigma$. Therefore we get

Theorem 3.3. If $\lambda \neq 0,-1$, then

$$
\operatorname{Der\overline {\mathfrak {g}}}=\operatorname{span}_{\mathbb{C}}\left\{\bar{\varphi}, \bar{\psi}, \overline{\sigma_{(-2)}}, \overline{\xi_{\mathcal{A}}}, \overline{\eta_{\mathcal{A}, 1}}, a d L_{a}, a d I_{a} \mid \mathcal{A} \in \operatorname{Hom}(G, \mathbb{C}), a \in G\right\},
$$

where $\varphi, \psi, \sigma_{(-2)}, \xi_{\mathcal{A}}, \eta_{\mathcal{A}, 1}$ are as in Theorem 3.1 and $\bar{\varphi}, \bar{\psi}, \overline{\sigma_{(-2)}}, \overline{\xi_{\mathcal{A}}}, \overline{\eta_{\mathcal{A}, 1}}$ defined in (3.10).

Now we consider the $\lambda=0$ case. We emphasize again that $\overline{\mathfrak{g}}$ is the higher rank Heisenberg-Virasoro algebra in this case. There are four kinds of derivations $\varphi, \psi, \sigma_{(0)}, \xi_{\mathcal{A}}$, which are of degree 0.

Theorem 3.4. Let $\lambda=0$ and $\mathcal{A} \in \operatorname{Hom}(G, \mathbb{C})$ (here we denote $C_{L I}=C_{L I}^{(0)}$ ).

(1) The derivation $\varphi$ lifts uniquely to a derivation $\bar{\varphi}$ of $\overline{\mathfrak{g}}$ defined by

$$
L_{a} \mapsto a I_{a}+\delta_{a, 0} C_{L I}, I_{a} \mapsto C_{I} \delta_{a, 0}, C_{L} \mapsto-24 C_{L I}, C_{L I} \mapsto C_{I}, C_{I} \mapsto 0
$$

(2) The derivation $\sigma_{(0)}$ lifts uniquely to a derivation $\overline{\sigma_{(0)}}$ of $\overline{\mathfrak{g}}$ defined by

$$
L_{a} \mapsto I_{a}-\delta_{a, 0} C_{L I}, I_{a} \mapsto-C_{I} \delta_{a, 0}, C_{L} \mapsto 0, C_{L I} \mapsto 0, C_{I} \mapsto 0
$$

(3) The derivation $\xi_{\mathcal{A}}$ lifts to a family of derivations $\left\{\overline{\xi_{\mathcal{A}, l, k}} \mid l, k \in \mathbb{C}\right\}$ of $\overline{\mathfrak{g}}$ defined by

$$
\begin{aligned}
& L_{a} \mapsto \mathcal{A}(a) L_{a}+(l+k a) I_{a}+\delta_{a, 0}(k-l) C_{L I}, \\
& I_{a} \mapsto \mathcal{A}(a) I_{a}-\delta_{a, 0} C_{I}(l+k), \\
& C_{L} \mapsto-24 k C_{L I}, C_{L I} \mapsto-k C_{I}, C_{I} \mapsto 0 .
\end{aligned}
$$

(4) The derivation $\psi$ lifts to a family of derivations $\left\{\overline{\psi_{l, k}} \mid l, k \in \mathbb{C}\right\}$ of $\overline{\mathfrak{g}}$ defined by

$$
\begin{aligned}
& L_{a} \mapsto(l+k a) I_{a}+\delta_{a, 0}(k-l) C_{L I}, \\
& I_{a} \mapsto I_{a}+\delta_{a, 0} C_{I}(k-l), \\
& C_{L} \mapsto-24 k C_{L I}, C_{L I} \mapsto C_{L I}+k C_{I}, C_{I} \mapsto 2 C_{I} .
\end{aligned}
$$

Proof. We only prove (3), the other three are similar and we omit it.

Let $\phi$ be a lift of $\xi_{\mathcal{A}}$. Notice that $\phi$ is homogeneous of degree 0 . We may write

$$
\phi\left(L_{a}\right)=\mathcal{A}(a) L_{a}+f_{L}(a) I_{a}+\delta_{a, 0} C_{1} ; \quad \phi\left(I_{a}\right)=f_{I}(a) L_{a}+\mathcal{A}(a) I_{a}+\delta_{a, 0} C_{2},
$$


for some $C_{1}, C_{2} \in \mathfrak{c}$ and functions $f_{L}, f_{I}: G \longrightarrow \mathbb{C}$. Expanding the equation $\phi\left(\left[L_{a}, L_{b}\right]\right)=$ $\left[\phi\left(L_{a}\right), L_{b}\right]+\left[L_{a}, \phi\left(L_{b}\right)\right]$ and comparing coefficients we get $(b-a) f_{L}(a+b)=b f_{L}(b)-a f_{L}(a)$, which implies

$$
f_{L}(a)=l+k a \text { for some } l, k \in \mathbb{C},
$$

and moreover we get

$$
\frac{1}{12}\left(a^{3}-a\right) \phi\left(C_{L}\right)-2 a C_{1}=\left(f_{L}(-a)\left(a^{2}+a\right)-f_{L}(a)\left(a^{2}-a\right)\right) C_{L I}=2 a\left(l-k a^{2}\right) C_{L I} .
$$

Let $a=1$, we see $C_{1}=(k-l) C_{L I}$. Hence $\phi\left(C_{L}\right)=-24 k C_{L I}$.

Expanding $\phi\left(\left[L_{a}, I_{b}\right]\right)=\left[\phi\left(L_{a}\right), I_{b}\right]+\left[L_{a}, \phi\left(I_{b}\right)\right]$ we get $b f_{I}(a+b)=f_{I}(b)(b-a)$, which implies $f_{I}(a)=0$ for any $a \in G$, and we get

$$
\left(a^{2}+a\right) \phi\left(C_{L I}\right)-a C_{2}=a(l-k) C_{I} .
$$

Let $a=-1$ and we see that $C_{2}=-(l+k) C_{I}, \phi\left(C_{L I}\right)=-k C_{I}$.

At last expand $\phi\left(\left[I_{a}, I_{b}\right]\right)$ and we have $\phi\left(C_{I}\right)=0$. This proves (3).

\section{Isomorphism classes and automorphisms}

In this section we determine the isomorphism classes and automorphism groups of the Lie algebras $\mathfrak{g}(G, \lambda)$ for arbitrary $G$ and $\lambda$, and then consider the lifts of these automorphisms to automorphisms of $\overline{\mathfrak{g}}$ for $\lambda \neq-1$ and free $G$.

Theorem 4.1. The Lie algebras $\mathfrak{g}(G, \lambda)$ and $\mathfrak{g}\left(G^{\prime}, \lambda^{\prime}\right)$ are isomorphic if and only if $\lambda^{\prime}=\lambda$ and $G^{\prime}=\xi G$ for some nonzero $\xi \in \mathbb{C}$. Moreover, any Lie algebra isomorphism $\pi$ : $\mathfrak{g}(G, \lambda) \longrightarrow \mathfrak{g}(\xi G, \lambda)$, aside from an inner automorphism of $\mathfrak{g}(\xi G, \lambda)$, has the form

$$
\begin{aligned}
& \pi\left(L_{a}\right)=\xi^{-1} \chi(a) L_{\xi a}^{\prime}+\chi(a) I_{\xi a}^{\prime}\left(l_{0} \delta_{\lambda, 0}+l_{1} a+l_{2} a^{2} \delta_{\lambda,-1}+l_{3} a^{3} \delta_{\lambda,-2}+f(a) \delta_{\lambda, 1}\right) ; \\
& \pi\left(I_{a}\right)=l \chi(a) I_{\xi a}^{\prime},
\end{aligned}
$$

where $l \in \mathbb{C}^{\times}, l_{0}, l_{1}, l_{2}, l_{3} \in \mathbb{C}, \chi \in G^{*}$ is a character of $G$, and $f \in \operatorname{Hom}(G, \mathbb{C})$.

Proof. Denote $\mathfrak{g}=\mathfrak{g}(G, \lambda), \mathfrak{g}^{\prime}=\mathfrak{g}\left(G^{\prime}, \lambda^{\prime}\right)$, and we will use an extra dash to denote elements in $\mathfrak{g}^{\prime}$ in the following. If $\lambda^{\prime}=\lambda, G^{\prime}=\xi G$ for some nonzero $\xi \in \mathbb{C}$, it is easy to check that the linear map defined by

$$
L_{a} \mapsto \xi^{-1} L_{\xi a}^{\prime} ; \quad I_{a} \mapsto \xi^{-1} I_{\xi a}^{\prime}
$$

is a Lie algebra isomorphism from $\mathfrak{g}$ to $\mathfrak{g}^{\prime}$.

On the other hand, suppose $\mathfrak{g} \cong \mathfrak{g}^{\prime}$ and let $\pi: \mathfrak{g} \longrightarrow \mathfrak{g}^{\prime}$ be a Lie algebra isomorphism.

Claim 1: There exists some $\xi \in \mathbb{C}^{\times}$and an inner automorphism $\theta$ of $\mathfrak{g}^{\prime}$ such that $G^{\prime}=\xi G$ and $\theta \pi\left(\mathfrak{g}_{a}\right)=\mathfrak{g}_{\xi a}^{\prime}$ for any $a \in G$.

Notice that the set of locally finite elements in $\mathfrak{g}$ is $\operatorname{span}_{\mathbb{C}}\left\{L_{0}, I_{a} \mid a \in G\right\}$. Since $\pi$ maps a locally finite element in $\mathfrak{g}$ to a locally finite element in $\mathfrak{g}^{\prime}$, we may assume that

$$
\pi\left(L_{0}\right)=\xi^{-1} L_{0}^{\prime}+\sum_{b \in G^{\prime}\left(L_{0}\right)} \gamma_{b} I_{b}^{\prime}
$$


for some $\xi \in \mathbb{C}^{\times}$, finite subset $G^{\prime}\left(L_{0}\right)$ of $G^{\prime}$ and $\gamma_{b} \in \mathbb{C}$. Define

$$
\eta=\prod_{0 \neq b \in G^{\prime}\left(L_{0}\right)} \exp \left\{-\frac{\xi \gamma_{b}}{b} \operatorname{ad} I_{b}^{\prime}\right\}
$$

which is an inner automorphism of $\mathfrak{g}^{\prime}$. Clearly, $\eta \pi\left(L_{0}\right)=\xi^{-1} L_{0}^{\prime}+\gamma_{0} I_{0}^{\prime}$. So replacing $\pi$ by $\eta^{-1} \pi$ we may assume

$$
\pi\left(L_{0}\right)=\xi^{-1} L_{0}^{\prime}+\gamma_{0} I_{0}^{\prime}
$$

Since $\operatorname{span}_{\mathbb{C}}\left\{I_{a} \mid a \in G\right\}$ is the unique maximal abelian ideal of $\mathfrak{g}$, it forces

$$
\pi\left(I_{a}\right)=\sum_{b \in G^{\prime}\left(I_{a}\right)} \nu_{a}(b) I_{b}^{\prime}
$$

for some finite subset $G^{\prime}\left(I_{a}\right)$ of $G^{\prime}$ and some function $\nu_{a}: G^{\prime} \longrightarrow \mathbb{C}$.

For any $a \neq 0$, from

$$
\pi\left[L_{0}, I_{a}\right]=a \sum_{b \in G^{\prime}\left(I_{a}\right)} \nu_{a}(b) I_{b}^{\prime}=\left[\pi\left(L_{0}\right), \pi\left(I_{a}\right)\right]=\xi^{-1} \sum_{b \in G^{\prime}\left(I_{a}\right)} b \nu_{a}(b) I_{b}^{\prime}
$$

we see that $G^{\prime}\left(I_{a}\right)=\{\xi a\} \subset G^{\prime}$ for any $a \neq 0$. This implies $G^{\prime}=\xi G$ and

$$
\pi\left(I_{a}\right)=\nu_{a}(\xi a) I_{\xi a}^{\prime} \text { for } a \neq 0 .
$$

Since $\pi\left[L_{a}, I_{b}\right]=(b-\lambda a) \pi\left(I_{a+b}\right)=(b-\lambda a) \nu_{a+b}(\xi(a+b)) I_{\xi(a+b)}^{\prime}=\left[\pi\left(L_{a}\right), \nu_{b}(\xi b) I_{\xi b}^{\prime}\right]$ for $a, b \neq 0, a+b \neq 0$, it follows that $\pi\left(L_{a}\right), a \neq 0$, must have the form

$$
\pi\left(L_{a}\right)=\rho(a) L_{\xi a}^{\prime}+\sum_{c \in G^{\prime}\left(L_{a}\right)} \mu(c) I_{c}^{\prime}
$$

for some finite subset $G^{\prime}\left(L_{a}\right)$ of $G^{\prime}$, functions $\rho: G \longrightarrow \mathbb{C}^{\times}$and $\mu: G^{\prime} \longrightarrow \mathbb{C}$. Then

$$
(b-\lambda a) \nu_{a+b}(\xi(a+b)) I_{\xi(a+b)}^{\prime}=\left[\rho(a) L_{\xi a}^{\prime}, \nu_{b}(\xi b) I_{\xi b}^{\prime}\right]=\xi \rho(a) \nu_{b}(\xi b)\left(b-\lambda^{\prime} a\right) I_{\xi(a+b)}^{\prime} .
$$

Moreover, from

$$
\pi\left[L_{a}, I_{0}\right]=-\lambda a \pi\left(I_{a}\right)=-\lambda a \nu_{a}(\xi a) I_{\xi a}^{\prime}=\left[\rho(a) L_{\xi a}^{\prime}, \sum_{b \in G^{\prime}\left(I_{0}\right)} \nu_{0}(b) I_{b}^{\prime}\right]
$$

we see that $G^{\prime}\left(I_{0}\right)=\{0\} \subset G^{\prime}$, and hence $\pi\left(I_{0}\right)=\nu_{0}(0) I_{0}^{\prime}$. Define

$$
\nu(\xi b)=\nu_{b}(\xi b) \text { for any } b \in G .
$$

We get a function $\nu: G^{\prime} \longrightarrow \mathbb{C}^{\times}$such that

$$
\pi\left(I_{a}\right)=\nu(\xi a) I_{\xi a}^{\prime} \in \mathfrak{g}_{\xi a}^{\prime} \quad \text { for any } a \in G .
$$

Then (4.2) turns to

$$
(b-\lambda a) \nu(\xi(a+b))=\xi \rho(a) \nu(\xi b)\left(b-\lambda^{\prime} a\right) .
$$


Consider $\pi\left[L_{0}, L_{a}\right]$ for $a \neq 0$, we get

$$
\sum_{b \in G^{\prime}\left(L_{a}\right)} \mu(b)\left(a-\xi^{-1} b\right) I_{b}^{\prime}-\gamma_{0} \lambda^{\prime} \xi a \rho(a) I_{\xi a}^{\prime}=0,
$$

which implies $G^{\prime}\left(L_{a}\right)=\{\xi a\}$ and $\lambda^{\prime} \gamma_{0}=0$. Write $\mu(0)=\gamma_{0}$ and $\rho(0)=\xi^{-1}$. Then we have

$$
\pi\left(L_{a}\right)=\rho(a) L_{\xi a}^{\prime}+\mu(\xi a) I_{\xi a}^{\prime} \in \mathfrak{g}_{\xi a}^{\prime} \quad \text { for any } a \in G .
$$

This proves Claim 1.

In the following we determine the functions $\rho, \mu, \nu$.

Claim 2: $\chi=\xi \rho$ is a character of $G, \lambda^{\prime}=\lambda$ and $\nu(\xi a)=\nu(0) \chi(a)$ for any $a \in G$. Since

$$
\begin{aligned}
& \pi\left[L_{a}, L_{b}\right]=(b-a) \pi\left(L_{a+b}\right)=(b-a) \rho(a+b) L_{\xi(a+b)}^{\prime}+(b-a) \mu(\xi(a+b)) I_{\xi(a+b)}^{\prime} \\
& =\left[\rho(a) L_{\xi a}^{\prime}+\mu(\xi a) I_{\xi a}^{\prime}, \rho(b) L_{\xi b}^{\prime}+\mu(\xi b) I_{\xi b}^{\prime}\right] \\
& =\xi(b-a) \rho(a) \rho(b) L_{\xi(a+b)}^{\prime}+\rho(a) \mu(\xi b) \xi\left(b-\lambda^{\prime} a\right) I_{\xi(a+b)}^{\prime}-\rho(b) \mu(\xi a) \xi\left(a-\lambda^{\prime} b\right) I_{\xi(a+b)}^{\prime},
\end{aligned}
$$

we get

$$
\begin{aligned}
& (b-a)(\rho(a+b)-\xi \rho(a) \rho(b))=0, \\
& (b-a) \mu(\xi(a+b))=\xi \rho(a) \mu(\xi b)\left(b-\lambda^{\prime} a\right)-\xi \rho(b) \mu(\xi a)\left(a-\lambda^{\prime} b\right) .
\end{aligned}
$$

From (4.4) we get that if $a \neq b$ then

$$
\rho(a+b)=\xi \rho(a) \rho(b) .
$$

Let $b=0$ in (4.6), we have $\rho(0)=\xi^{-1}$. Choose $b \neq 0, \pm a$. Using (4.6) we get

$$
\rho(2 a)=\xi \rho(a+b) \rho(a-b)=\xi^{3} \rho(a)^{2} \rho(b) \rho(-b)=\xi^{2} \rho(a)^{2} \rho(0)=\xi \rho(a)^{2} \text { for any } a \in G .
$$

This proves that (4.6) stands for any $a, b \in G$. So $\chi=\xi \rho$ is a character of $G$.

Let $b=0$ in (4.3) we get

$$
\lambda \nu(\xi a)=\lambda^{\prime} \xi \nu(0) \rho(a) \text { for any } a \neq 0,
$$

which implies that

$$
\lambda^{\prime}=0 \text { if and only if } \lambda=0 .
$$

If $\lambda \neq 0$, then (4.7) says $\nu(\xi a)=\frac{\lambda^{\prime} \xi}{\lambda} \nu(0) \rho(a)$ for any $a \neq 0$. Putting it into (4.3) and using (4.6) we see $\lambda^{\prime}=\lambda$. Hence $\nu(\xi a)=\xi \nu(0) \rho(a)=\nu(0) \chi(a)$ if $\lambda \neq 0$.

If $\lambda=\lambda^{\prime}=0$, let $a=-b \neq 0$ in (4.3) and we have

$$
\nu(0)=\xi \rho(-b) \nu(\xi b)
$$

Then $\nu(0) \chi(b)=\xi^{2} \rho(b) \rho(-b) \nu(\xi b)=\xi \rho(0) \nu(\xi b)=\nu(\xi b)$. This proves Claim 2 .

At last we determine the function $\mu$. Set $\varphi(a)=\frac{\mu(\xi a)}{\chi(a)}$. Divide $\chi(a+b)$ to (4.5) and we obtain

$$
(b-a) \varphi(a+b)+(a-\lambda b) \varphi(a)=(b-\lambda a) \varphi(b),
$$


which is the same equation as (3.4). So from the computation in Section 3 we have

$$
\varphi(a)=l_{0} \delta_{\lambda, 0}+l_{1} a+l_{2} a^{2} \delta_{\lambda,-1}+l_{3} a^{3} \delta_{\lambda,-2}+f(a) \delta_{\lambda, 1},
$$

where $l_{0}, l_{1}, l_{2}, l_{3} \in \mathbb{C}$ and $f \in \operatorname{Hom}(G, \mathbb{C})$. Therefore

$$
\mu(\xi a)=\chi(a)\left(l_{0} \delta_{\lambda, 0}+l_{1} a+l_{2} a^{2} \delta_{\lambda,-1}+l_{3} a^{3} \delta_{\lambda,-2}+f(a) \delta_{\lambda, 1}\right) .
$$

This proves the theorem.

Now using Theorem 4.1 we may determine the automorphism group of $\mathfrak{g}$. Denote $\mathcal{E}=$ $\left\{\epsilon \in \mathbb{C}^{\times} \mid \epsilon G=G\right\}$, which is a subgroup of $\mathbb{C}^{\times}$. Let $\xi \in \mathcal{E}, \chi \in G^{*}, f \in \operatorname{Hom}(G, \mathbb{C}), l \in$ $\mathbb{C}^{\times}, l_{0}, l_{1}, l_{2}, l_{3} \in \mathbb{C}$, denote by $\theta_{\lambda}\left(\xi, \chi, f, l, l_{0}, l_{1}, l_{2}, l_{3}\right)$ the linear map given by

$$
\begin{aligned}
& L_{a} \mapsto \xi^{-1} \chi(a) L_{\xi a}+\chi(a) I_{\xi a}\left(l_{0} \delta_{\lambda, 0}+l_{1} a+l_{2} a^{2} \delta_{\lambda,-1}+l_{3} a^{3} \delta_{\lambda,-2}+f(a) \delta_{\lambda, 1}\right) \\
& I_{a} \mapsto l \chi(a) I_{\xi a} .
\end{aligned}
$$

Here we shall point out that if $\lambda=1$ we may assume $l_{1}=0$ since the map $a \mapsto f(a)+l_{1} a$ still lies in $\operatorname{Hom}(G, \mathbb{C})$. Then by Theorem 4.1 we have

Theorem 4.2. The outer automorphism group of $\mathfrak{g}$ is

$$
\text { Outg }=\left\{\theta_{\lambda}\left(\xi, \chi, f, l, l_{0}, l_{1}, l_{2}, l_{3}\right) \mid \xi \in \mathcal{E}, \chi \in G^{*}, f \in \operatorname{Hom}(G, \mathbb{C}), l \in \mathbb{C}^{\times}, l_{0}, l_{1}, l_{2}, l_{3} \in \mathbb{C}\right\},
$$

and $A u t \mathfrak{g}=$ Outg $\ltimes$ Inng $\mathfrak{g}$, where Inng is the inner automorphism group of $\mathfrak{g}$, generated by $\left\{\exp a d I_{a} \mid a \in G\right\}$.

Notice that

$$
\begin{aligned}
& \theta_{\lambda}\left(\xi^{\prime}, \chi^{\prime}, f^{\prime}, l^{\prime}, l_{0}^{\prime}, l_{1}^{\prime}, l_{2}^{\prime}, l_{3}^{\prime}\right) \cdot \theta_{\lambda}\left(\xi, \chi, f, l, l_{0}, l_{1}, l_{2}, l_{3}\right) \\
& =\theta_{\lambda}\left(\xi^{\prime} \xi,\left(\chi^{\prime} \cdot \xi\right) \chi, f^{\prime}+l^{\prime} f, l^{\prime} l, \xi^{-1} l_{0}^{\prime}+l^{\prime} l_{0}, l_{1}^{\prime}+l^{\prime} l_{1}, \xi l_{2}^{\prime}+l^{\prime} l_{2}, \xi^{2} l_{3}^{\prime}+l^{\prime} l_{3}\right)
\end{aligned}
$$

and

$$
\begin{aligned}
& \theta_{\lambda}\left(\xi, \chi, f, l, l_{0}, l_{1}, l_{2}, l_{3}\right)^{-1}= \\
& \quad \theta_{\lambda}\left(\xi^{-1}, \chi^{-1} \cdot \xi^{-1},-l^{-1} f, l^{-1},-l^{-1} \xi l_{0},-l^{-1} l_{1},-l^{-1} \xi^{-1} l_{2},-l^{-1} \xi^{-2} l_{3}\right) .
\end{aligned}
$$

Clearly, the map $G^{*} \longrightarrow$ Outg defined by $\chi \mapsto \theta_{\lambda}(1, \chi, 0,1,0,0,0,0)$ is a group monomorphism and denote by $N$ its image. Set $S=\left\{\theta_{\lambda}(1, \mathbb{1}, 0, l, 0,0,0,0) \mid l \in \mathbb{C}^{\times}\right\}$, and

$$
K=\left\{\theta_{\lambda}\left(1, \mathbb{1}, f, 1, l_{0}, l_{1}, l_{2}, l_{3}\right) \mid l_{0}, l_{1}, l_{2}, l_{3} \in \mathbb{C}, f \in \operatorname{Hom}(G, \mathbb{C})\right\} .
$$

Here $\mathbb{1}$ denotes the identity in $G^{*}$. Clearly, $N, S, K$ are normal subgroups of Outg, and

$$
N \cong G^{*}, S \cong \mathbb{C}^{\times}, \text {and } K \cong \begin{cases}\operatorname{Hom}(G, \mathbb{C}) \cong \mathbb{C}^{n} & \text { if } \lambda=1 \\ \mathbb{C}^{2} & \text { if } \lambda=0,-1,-2 \\ \mathbb{C} & \text { otherwise }\end{cases}
$$

Moreover, let $T=\left\{\theta_{\lambda}(\xi, \mathbb{1}, 0,1,0,0,0,0) \mid \xi \in \mathcal{E}\right\}$, which is a subgroup of Outg and isomorphic to $\mathcal{E}$. Then we have a projection Outg $\longrightarrow T$, whose kernel is the normal subgroup $N S K$. So we get 
Theorem 4.3. Outg $=T \ltimes(N S K) \cong \mathcal{E} \ltimes\left(G^{*} \mathbb{C}^{\times} K\right)$.

Using a result from [10] one may extend automorphisms of $\mathfrak{g}$ to automorphisms of $\overline{\mathfrak{g}}$.

Proposition $4.4([10])$. Let $\mathcal{G}$ be a perfect Lie algebra and $\overline{\mathcal{G}}$ the universal central extension of $\mathcal{G}$. Then every automorphism $\theta$ of $\mathfrak{g}$ admits a unique extension to an automorphism $\bar{\theta}$ of $\overline{\mathcal{G}}$, and the map $\theta \mapsto \bar{\theta}$ is a group monomorphism. Moreover, if $\mathcal{G}$ is centerless, then the map $\theta \mapsto \bar{\theta}$ is an isomorphism.

Explicitly we have the form of extended automorphisms.

Theorem 4.5. Suppose $\lambda \neq-1$. Let $\xi \in \mathcal{E}, \chi \in G^{*}, f \in \operatorname{Hom}(G, \mathbb{C}), l \in \mathbb{C}^{\times}, l_{0}, l_{1}, l_{3} \in \mathbb{C}$, then the unique automorphism $\bar{\theta}=\overline{\theta_{\lambda}\left(\xi, \chi, f, l, l_{0}, l_{1}, 0, l_{3}\right)}$ of $\overline{\mathfrak{g}}$ obtained by extension from $\theta=\theta_{\lambda}\left(\xi, \chi, f, l, l_{0}, l_{1}, 0, l_{3}\right)$ is such that

$$
\begin{gathered}
\bar{\theta}\left(L_{a}\right)=\xi^{-1} \chi(a) L_{\xi a}+\chi(a) I_{\xi a}\left(l_{0} \delta_{\lambda, 0}+l_{1} a+l_{3} a^{3} \delta_{\lambda,-2}+f(a) \delta_{\lambda, 1}\right) \\
\quad+\delta_{a, 0}\left(\frac{\xi^{-1}-\xi}{24} C_{L}+\left(l_{1} \xi-l_{0}\right) C_{L I}^{(0)} \delta_{\lambda, 0}+\frac{1}{2} \xi\left(l_{1}^{2}-l_{0}^{2}\right) C_{I} \delta_{\lambda, 0}\right) ; \\
\bar{\theta}\left(I_{a}\right)=l \chi(a) I_{\xi a}+l \xi \delta_{a, 0}\left(\frac{\xi^{-1}-\xi}{24} C_{L I}^{(1)} \delta_{\lambda, 1}+\left(\left(1-\xi^{-1}\right) C_{L I}^{(0)}+\left(l_{1}-l_{0}\right) C_{I}\right) \delta_{\lambda, 0}\right) \\
\quad+l\left(\xi \epsilon_{1}\right)^{-1} \delta_{a, 0} \sum_{i=2}^{n}\left(\xi \epsilon_{1}\right)_{i} C_{L I}^{(i)} \delta_{\lambda,-2} ; \\
\bar{\theta}\left(C_{L}\right)=\xi C_{L}-12 l_{1} \xi\left(2 C_{L I}^{(0)}+l_{1} C_{I}\right) \delta_{\lambda, 0} ; \\
\bar{\theta}\left(C_{L I}^{(0)}\right)=l \xi\left(C_{L I}^{(0)}+l_{1} C_{I}\right) ; \quad \bar{\theta}\left(C_{L I}^{(1)}\right)=l \xi^{2} C_{L I}^{(1)} ; \\
\bar{\theta}\left(C_{L I}^{(i)}\right)=l\left(\xi \epsilon_{1}\right)^{-1} \sum_{j=2}^{n}\left\{\epsilon_{1}\left(\xi \epsilon_{i}\right)_{j}-\epsilon_{i}\left(\xi \epsilon_{1}\right)_{j}\right\} C_{L I}^{(j)}, i \geq 2 ; \\
\bar{\theta}\left(C_{I}\right)=l^{2} \xi C_{I} .
\end{gathered}
$$

Here for $a \in G$ we use $a_{i}$ to denote the coefficients of a with respect to the basis $\epsilon_{1}, \ldots, \epsilon_{n}$.

Proof. Recall $\mathfrak{c}=\operatorname{span}_{\mathbb{C}}\left\{C_{L}, C_{I} \delta_{\lambda, 0}, C_{L I}^{(0)} \delta_{\lambda, 0}, C_{L I}^{(1)} \delta_{\lambda, 1}, C_{L I}^{(i)} \delta_{\lambda,-2} \mid 2 \leq i \leq n\right\}$. For later convenience, we denote

$$
\begin{aligned}
& \tau(a)=l_{0} \delta_{\lambda, 0}+l_{1} a+l_{3} a^{3} \delta_{\lambda,-2}+f(a) \delta_{\lambda, 1}, \\
& \nu_{C}(a)=C_{L I}^{(0)}\left(a^{2}+a\right) \delta_{\lambda, 0}+\frac{1}{12}\left(a^{3}-a\right) C_{L I}^{(1)} \delta_{\lambda, 1}+\sum_{i=2}^{n} a_{i} C_{L I}^{(i)} \delta_{\lambda,-2} \in \mathfrak{c} .
\end{aligned}
$$

Clearly, $\bar{\theta}\left(L_{a}\right)$ and $\bar{\theta}\left(I_{a}\right)$ have the form

$$
\begin{aligned}
\bar{\theta}\left(L_{a}\right) & =\xi^{-1} \chi(a) L_{\xi a}+\chi(a) \tau(a) I_{\xi a}+K_{L}(a) ; \\
\bar{\theta}\left(I_{a}\right) & =l \chi(a) I_{\xi a}+K_{I}(a),
\end{aligned}
$$


for some maps $K_{L}, K_{I}: G \longrightarrow \mathfrak{c}$. Expanding

$$
\bar{\theta}\left(\left[L_{a}, L_{b}\right]\right)=\left[\xi^{-1} \chi(a) L_{\xi a}+\chi(a) \tau(a) I_{\xi a}, \xi^{-1} \chi(b) L_{\xi b}+\chi(b) \tau(b) I_{\xi b}\right]
$$

we see that $K_{L}(a)=0$ if $a \neq 0$, and

$$
-2 K_{L}(0)+\frac{1}{12}\left(a^{2}-1\right) \bar{\theta}\left(C_{L}\right)=\frac{\xi a^{2}-\xi^{-1}}{12} C_{L}+2\left(l_{0}-l_{1} \xi a^{2}\right) C_{L I}^{(0)} \delta_{\lambda, 0}+\left(l_{0}^{2}-l_{1}^{2} a^{2}\right) \xi C_{I} \delta_{\lambda, 0},
$$

which implies

$$
K_{L}(0)=\frac{\xi^{-1}-\xi}{24} C_{L}+\left(l_{1} \xi-l_{0}\right) C_{L I}^{(0)} \delta_{\lambda, 0}+\frac{1}{2} \xi\left(l_{1}^{2}-l_{0}^{2}\right) C_{I} \delta_{\lambda, 0}
$$

and

$$
\bar{\theta}\left(C_{L}\right)=\xi C_{L}-12 l_{1} \xi\left(2 C_{L I}^{(0)}+l_{1} C_{I}\right) \delta_{\lambda, 0} .
$$

This proves (4.8) and (4.10).

Expanding $\bar{\theta}\left(\left[L_{a}, I_{b}\right]\right)=\left[\bar{\theta}\left(L_{a}\right), \bar{\theta}\left(I_{b}\right)\right]$ we get that $K_{I}(a)=0$ for $a \neq 0$ and

$$
\begin{aligned}
& -(1+\lambda) a K_{I}(0)+\left(a^{2}+a\right) \bar{\theta}\left(C_{L I}^{(0)}\right) \delta_{\lambda, 0}+\frac{a^{3}-a}{12} \bar{\theta}\left(C_{L I}^{(1)}\right) \delta_{\lambda, 1}+\sum_{i=2}^{n} a_{i} \bar{\theta}\left(C_{L I}^{(i)}\right) \delta_{\lambda,-2} \\
& =l \xi^{-1}\left(\left((\xi a)^{2}+\xi a\right) C_{L I}^{(0)} \delta_{\lambda, 0}+\frac{(\xi a)^{3}-\xi a}{12} C_{L I}^{(1)} \delta_{\lambda, 1}+\xi \sum_{i=2}^{n} a_{i} C_{L I}^{(i)} \delta_{\lambda,-2}\right)+l \tau(a) \xi a C_{I} \delta_{\lambda, 0} .
\end{aligned}
$$

If $\lambda=0$, let $a=-1$ in (4.13), then we get

$$
K_{I}(0) \delta_{\lambda, 0}=l \xi\left(\left(1-\xi^{-1}\right) C_{L I}^{(0)}+\left(l_{1}-l_{0}\right) C_{I}\right) \delta_{\lambda, 0} \text { and } \bar{\theta}\left(C_{L I}^{(0)}\right)=l \xi\left(C_{L I}^{(0)}+l_{1} C_{I}\right) .
$$

If $\lambda=1$, let $a=1$ in (4.13), then we get

$$
K_{I}(0) \delta_{\lambda, 1}=-\frac{l\left(\xi^{2}-1\right)}{24} C_{L I}^{(1)} \delta_{\lambda, 1} \text { and } \bar{\theta}\left(C_{L I}^{(1)}\right)=l \xi^{2} C_{L I}^{(1)} .
$$

If $\lambda=-2$, let $0 \neq a \in \mathbb{Z} \epsilon_{1}$ in (4.13), then we get

$$
\begin{aligned}
& K_{I}(0) \delta_{\lambda,-2}=l\left(\xi \epsilon_{1}\right)^{-1} \sum_{i=2}^{n}\left(\xi \epsilon_{1}\right)_{i} C_{L I}^{(i)} \delta_{\lambda,-2} \\
& \bar{\theta}\left(C_{L I}^{(i)}\right)=l\left(\xi \epsilon_{1}\right)^{-1} \sum_{j=2}^{n}\left(\epsilon_{1}\left(\xi \epsilon_{i}\right)_{j}-\epsilon_{i}\left(\xi \epsilon_{1}\right)_{j}\right) C_{L I}^{(j)} .
\end{aligned}
$$

This proves (4.9) and (4.11). Similarly, (4.12) follows from $\bar{\theta}\left(\left[I_{a}, I_{b}\right]\right)=\left[\bar{\theta}\left(I_{a}\right), \bar{\theta}\left(I_{b}\right)\right]$. 


\section{References}

[1] E. Arbarello, C. De Concini, V. G. Kac and C. Procesi, Moduli spaces of curves and representation theory, Comm. Math. Phys. 117(1)(1988) 1-36.

[2] G. M. Benkart and R. V. Moody, Derivations, central extensions and affine Lie algebras, Algebras Groups Geom. 3(4)(1986) 456-492.

[3] D. Z. Dokovic and K. Zhao, Derivations, isomorphisms and second cohomology of generalized Witt algebras, Trans. Amer. Math. Soc. 350(2)(1998) 643-664.

[4] R.Farnsteiner, Derivations and central extensions of finitely generated graded Lie algebras, J. Algebra 118(1)(1988) 33-45.

[5] D. Liu, Classification of Harish-Chandra modules over some Lie algebras related to the Virasoro algebra, J. Algebra 447(1)(2016) 548-559.

[6] G. Liu and X. Guo, Harish-Chandra modules over generalzied Heisenberg-Virasoro algebras, Israel J. Math. 204(1)(2014) 447-468.

[7] D. Liu and Y. Pei, Deformations on the twisted Heisenberg-Virasoro algebra, Chin. Ann. Math. Ser. B 40(1)(2019) 111-116.

[8] D. Liu and L. Zhu, Generalized Heisenberg-Virasoro algebras, Front. Math. China 4(2)(2009) 297-310.

[9] R. Lu and K. Zhao, Classification of irreducible weight modules over the twisted Heisenberg-Virasoro algebra, Commun. Contemp. Math. 12(2)(2010) 183C205.

[10] A. Pianzola, Automorphisms of toroidal Lie algebras and their central quotients, J. Algebra Appl. bf 1(1)(2002) 113-121.

[11] J. Patera and H. Zassenhaus, The higher rank Virasoro algebras, Commun. Math. Phys. 136(1)(1991) 1-14.

[12] S. Tan and X. Zhang, Automorphisms and Verma modules for generalized SchrodingerCVirasoro algebras, J. Algebra 322(1)(2009) 1379-1394.

[13] W. Wang, J. Li and B. Xin, Central extensions and derivations of generalized Schrodinger-Virasoro algebras, Algebra Colloq. 19(4)(2012) 735-744.

[14] C. Xu, Automorphisms, derivations and central extensions of Lie algebras arising from quantum tori, Comm. Algebra 47(10)(2019) 4009-4019. 